\title{
NOTE ON COMPLEMENTED MODULAR LATTICES
}

\author{
R. P. DILWORTH
}

1. Introduction. In this note we study those elements of a complemented modular lattice whose complements are unique. We show that these elements are simply the neutral ${ }^{1}$ elements of the lattice. It is also shown that an element with unique complement decomposes the lattice into a direct product of sublattices. ${ }^{2}$ Hence if the lattice is indecomposable, each element not the null or unit element must have at least two complements. In case the lattice is of finite dimensions these results give a new proof of the Birkhoff-Menger ${ }^{3}$ theorem that a complemented modular lattice of finite dimensions is a direct product of projective geometries and a Boolean algebra.

Although the existence of points and divisor-free elements is postulated, no chain conditions are assumed and the proofs are purely combinatorial.

2. Notation and definitions. Let $\subseteq$ denote a closed, complemented, modular lattice with null element $z$ and unit element $i$. Complements of $a \varepsilon \subseteq$ will be denoted by $a^{\prime}, a^{\prime \prime}, \ldots$ and have the property that $\left(a, a^{\prime}\right)=i,\left[a, a^{\prime}\right]=z$. We assume that each $a \neq z$ divides a point $p$, and that each $b \neq i$ is divisible by a divisor-free element $q$. It follows that $a \supset b, a \neq b$, implies the existence of a point $p \subset\left[a, b^{\prime}\right]$ and of a divisor-free element $q \supset\left(b, a^{\prime}\right)$ such that $a \supset p, b \ngtr p$ and $q \supset b, q \triangleright a$. Hence each element of $\subseteq$ is the union of the points which it divides and the crosscut of its divisor-free divisors.

If $S$ is a set of elements of $\subseteq, u(S)(k(S))$ will denote the union (crosscut) of the elements of $S$. If $a \varepsilon \subseteq$ we denote the set of points $p$ (divisor-free elements $q$ ) such that $a \supset p(q \supset a)$ by $P_{a}\left(Q_{a}\right)$. If $\subseteq$ is the direct product of the sublattices $\Im_{1}$ and $\widetilde{S}_{2}$, we write $\subseteq=\Im_{1} \times \widetilde{S}_{2}$.

An element $a$ of $\subseteq$ is said to be neutral if $(a,[b, c])=[(a, b),(a, c)]$ all $b, c \varepsilon \mathfrak{s}$. It is easily shown $n^{4}$ that $a$ is neutral if and only if $[a,(b, c)]$ $=([a, b],[a, c])$ all $b, c \varepsilon \mathfrak{S}$.

3. Properties of elements with unique complements. We need the following lemmas:

1 See $\$ 2$.

${ }^{2}$ Added in proof: Theorems 3.1 and 4.1 are given by J. von Neumann in his Continuous Geometrics (Princeton).

${ }^{3}$ Garrett Birkhoff, Annals of Mathematics, (2), vol. 36 (1935), pp. 743-748; K. Menger, Annals of Mathematics, (2), vol. 37 (1936), pp. 456-481. Professor Birkhoff has informed the author that he has also obtained Theorem 4.2.

4 O. Ore, Annals of Mathematics, (2), vol. 36 (1935), pp. 406-437. 
Lemma 3.1. If an element $a$ of $\subseteq$ has a unique complement $a^{\prime}$ and $a \supset b$, then $a^{\prime}=\left[b^{\prime},\left(b, a^{\prime}\right)\right]$.

Proof. It suffices to show that $\left[b^{\prime},\left(b, a^{\prime}\right)\right]$ is a complement of $a$. Now

$$
\left[a,\left[b^{\prime},\left(b, a^{\prime}\right)\right]\right]=\left[b^{\prime},\left[a,\left(b, a^{\prime}\right)\right]\right]=\left[b^{\prime},\left(b,\left[a, a^{\prime}\right]\right)\right]=\left[b, b^{\prime}\right]=z .
$$

Also

$$
\left(a,\left[b^{\prime},\left(b, a^{\prime}\right)\right]\right) \supset\left(b,\left[b^{\prime},\left(b, a^{\prime}\right)\right]\right)=\left[\left(b, b^{\prime}\right),\left(b, a^{\prime}\right)\right]=\left(b, a^{\prime}\right) \supset a^{\prime} .
$$

But $\left(a,\left[b^{\prime},\left(b, a^{\prime}\right)\right]\right) \supset a$. Hence $\left(a,\left[b^{\prime},\left(b, a^{\prime}\right)\right]\right) \supset\left(a, a^{\prime}\right)=i$.

LEMMA 3.2. If an element a of $\subseteq$ has a unique complement $a^{\prime}$ and if $p$ is a point of $\Im$, then either $a \supset p$ or $a^{\prime} \supset p$.

Proof. Suppose that $a \ngtr p$ and $a^{\prime} \ngtr p$. Let $p^{\prime}=\left[a,\left(a^{\prime}, p\right)\right]$. Then $p^{\prime}$ is a point and $p^{\prime} \neq p$ since otherwise $a \supset p$ contrary to assumption. Now let $q$ be a divisor-free element such that $q \ngtr p^{\prime}$ but $q \supset p$. (Such a $q$ always exists since $p=k\left(Q_{p}\right)$, and if $q \supset p^{\prime}$ all $q \varepsilon Q_{p}$, then $p \supset p^{\prime}$ and $p=p^{\prime}$, which is impossible.) Now since $q$ is a complement of $p^{\prime}$, we have $a^{\prime}=\left[q,\left(p^{\prime}, a^{\prime}\right)\right]$ by Lemma 3.1. But then

$$
\begin{aligned}
a^{\prime} & =\left[q,\left(p^{\prime}, a^{\prime}\right)\right]=\left[q,\left(a^{\prime},\left[a,\left(a^{\prime}, p\right)\right]\right)\right] \\
& =\left[q,\left(a^{\prime}, a\right),\left(a^{\prime}, p\right)\right]=\left[q,\left(a^{\prime}, p\right)\right]=\left(p,\left[q, q^{\prime}\right]\right) \supset p,
\end{aligned}
$$

contradicting $a^{\prime} \ngtr p$.

THEOREM 3.1. Let a have a unique complement $a^{\prime}$ and let $\mathfrak{a}\left(\mathfrak{a}^{\prime}\right)$ denote the sublattice of all elements $x(y)$ such that $a \supset x\left(a^{\prime} \supset y\right)$. Then $\mathfrak{S}=\mathfrak{a} \times \mathfrak{a}^{\prime}$.

Proof. We show first that $x=\left([a, x],\left[a^{\prime}, x\right]\right)$ any $x \varepsilon \mathfrak{S}$. By Lemma 3.2, $x=\left(u(S), u\left(S^{\prime}\right)\right)$ where $S\left(S^{\prime}\right)$ is the set of points divisible by $x$ and $a\left(a^{\prime}\right)$. Hence $S=P_{[a, x]}$ and $S^{\prime}=P_{\left[a^{\prime}, x\right]}$. Thus $x=([a, x]$, $\left.\left[a^{\prime}, x\right]\right)$. If $x=\left(x_{1}, x_{2}\right)$ where $a \supset x_{1}, a^{\prime} \supset x_{2}$, then $x_{1}=[a, x]$ and $x_{2}=\left[a^{\prime}, x\right]$ by the modular condition. Hence every element of $\subseteq$ is uniquely expressible as a union of elements of $\mathfrak{a}$ and $\mathfrak{a}^{\prime}$. Clearly $(x, y)=\left(\left(x_{1}, y_{1}\right),\left(x_{2}, y_{2}\right)\right)$ and $[x, y]=\left([a, x, y],\left[a^{\prime}, x, y\right]\right)=\left(\left[x_{1}, y_{1}\right]\right.$, $\left.\left[x_{2}, y_{2}\right]\right)$.

If $p_{1}$ and $p_{2}$ are distinct points, the element $\left(p_{1}, p_{2}\right)$ is called the "line" joining $p_{1}$ and $p_{2}$. We have then the following result:

TheOREm 3.2. An element $a \boldsymbol{\varepsilon} \mathfrak{\subseteq}$ has a unique complement $a^{\prime}$ if and only if every line joining points of $a$ and $a^{\prime}$ contains no other points.

Proof. Let $a$ have a unique complement $a^{\prime}$. Then $\subseteq=\mathfrak{a} \times \mathfrak{a}^{\prime}$ by 
Theorem 3.1. Let $a \supset p$ and $a^{\prime} \supset p^{\prime}$. If $\left(p, p^{\prime}\right) \supset p_{1}$ where $p_{1}$ is distinct from $p$ and $p^{\prime}$, then $a \triangleright p_{1}$ since otherwise $a \supset\left(p, p_{1}\right) \supset p^{\prime}$ and $\left[a, a^{\prime}\right] \supset p^{\prime}$, which is impossible. Similarly $a^{\prime} \ngtr p_{1}$. But this contradicts Lemma 3.2. On the other hand let $a$ have a second complement $a^{\prime \prime}$ and let $a^{\prime} \supset p^{\prime}, a^{\prime \prime} \gg p^{\prime}, a \supset p$ and $\left(a^{\prime \prime}, p^{\prime}\right) \supset p$. Then $p_{1}=\left[a^{\prime \prime},\left(p^{\prime}, p\right)\right]$ is a point distinct from $p$ and $p^{\prime}$. Clearly $\left(p, p^{\prime}\right) \supset p_{1}$.

The relationship of unique complements to projective geometry is clearly brought out by the following corollary to Theorem 3.2:

COROLlary. Every line of 5 contains at least three points if and only if each element not the null or unit element of $\subseteq$ has at least two complements.

If $\mathfrak{S}$ is of finite dimension, the Birkhoff-Menger representation theorem follows immediately from Theorems 3.1 and 3.2.

4. Distributivity. In this section we show that the elements with unique complements are the neutral elements of the lattice. We prove first a lemma:

Lemma 4.1. If a has a unique complement $a^{\prime}$, then $a$ is the unique complement of $a^{\prime}$.

Proof. Suppose that $a^{\prime}$ has a second complement $a_{1}$. Then $a \supset a_{1}$ by Theorem 3.1, and hence $a=a_{1}$ by the modular condition.

TheOREM 4.1. An element a has a unique complement if and only if it is neutral.

Proof. Let $a$ have a unique complement $a^{\prime}$. Then $[a,(b, c)]$ $=\left[a_{1},\left(b_{1}, c_{1}\right)\right]=\left[a,\left(b_{1}, c_{1}\right)\right]=\left(b_{1}, c_{1}\right)=([a, b],[a, c])$ by Theorem 3.1. Hence $a$ is neutral. Conversely let $a$ be neutral and let $a^{\prime}$ be a complement of $a$. Suppose that $a^{\prime}$ has a second complement $a_{1}$. Then $a=\left(a,\left[a_{1}, a^{\prime}\right]\right)=\left[\left(a, a_{1}\right),\left(a, a^{\prime}\right)\right]=\left(a, a_{1}\right)$ and hence $a \supset a_{1}$. Also $a=\left[a,\left(a_{1}, a^{\prime}\right)\right]=\left(\left[a, a_{1}\right],\left[a, a^{\prime}\right]\right)=\left[a, a_{1}\right]$ so that $a_{1} \supset a$. Hence $a=a_{1}$. Thus $a$ is the unique complement of $a^{\prime}$ and hence $a^{\prime}$ is the unique complement of $a$ by Lemma 4.1 .

It is interesting that Theorem 3.1 gives almost trivially the theorem that if $\mathfrak{S}$ is finite dimensional and complements are unique, then $\mathfrak{S}$ is a Boolean algebra.

California Institute of Technology 